\title{
Early life adversity induced third ventricular enlargement in young adult male patients suffered from major depressive disorder: a study of brain morphology
}

\author{
H. Zhao' ${ }^{1}$ T. Wei ${ }^{2}$, X. Li ${ }^{3}$, T. Ba ${ }^{2}$ \\ ${ }^{1}$ Department of Neurology, No. 261 Hospital of PLA, PR China \\ 2Department of Radiology, No. 261 Hospital of PLA, PR China \\ ${ }^{3}$ Department of Psychiatry III, No. 261 Hospital of PLA, PR China
}

[Received: 21 October 2017; Accepted: 30 November 2017]

\begin{abstract}
Background: Early life adversity (ELA) is not uncommon in major depressive disorder (MDD) patients. Childhood trauma has been reported more frequently in adult MDD patients relative to healthy controls. Recent researches have demonstrated that ELA could result in changes in brain morphology which might be an aetiological factor of MDD development.

Materials and methods: We recruited 40 young adult patients suffered from MDD and made computed tomography scan. Subjects were divided in two groups: MDD patients with ELA experience $(E+D)$ vs. MDD patients without ELA experience $(E-D)$ according to Chinese version-Childhood Trauma Questionnaire (CTQ). 17-item Hamilton Depression (HAMD) Scale and Neuropsychiatric Inventory (NPI) were also examined. Student's t-test was used to compare the HAMD scores, NPI scores, CTQ subcomponents scores, third ventricular (TV) width and volume of patients from $E+D$ and $E-D$ groups.

Results: Findings demonstrated that ELA might result in TV enlargement; furthermore, there was a correlation between physical neglect and TV volume.

Conclusions: These findings supported the hypothesis that ELA could induce changes of structure around the $T V$, which might undermine the aetiology of MDD. (Folia Morphol 2018; 77, 3: 428-433)
\end{abstract}

Key words: early life adversity, major depressive disorder, third ventricle, computed tomography scan

\section{INTRODUCTION}

Early life adversity (ELA) refers to series of adversities occurring before sexual maturation, including physical, sexual, and emotional abuse, physical and emotional neglect, malnourishment, and loss of a parent $[2,14]$. In Western countries, the experience of ELA is a common phenomenon. Up to $30-40 \%$ of the adult population report experiences of emotional or physical maltreatment during childhood [21]. In mainland China, the state of childhood maltreatment seems even severe. According to a study launched in 2004 , the prevalence of emotional abuse, physical abuse, and sexual abuse were $45.1 \%, 32.4 \%$, and $25.5 \%$, respectively [29].

Address for correspondence: Dr. T. Ba, 116 Zaojiatun, Shangzhuang town, Beijing, China, tel: +86 010-66346193, fax: +86 010-66346193, e-mail: Btr1959@163.com 
Early life adversity could be an important factor which affects the later lifestyle and personality [18]. For example, maltreatment before the age of 18 is associated with higher cigarette use during adolescence [17]. Adults reporting more adverse childhood experiences have poorer sleep quality compared with those having less or no adverse experiences [5]. In recent years, ELA was considered to be related to adult affective disorders. There is compelling evidence that early adverse experiences, such as parental loss and maltreatment, are associated with a higher risk for developing depression in later life [4, 9]. In a review, Heim et al. [13] revealed that ELA significantly increases the incidence of affective disorders and can have an impact on the development of emotional abilities.

Major depressive disorder (MDD) is a world-wide problem particularly affecting teenagers and young adults [24], and is among the most prevalent and burdensome of all psychiatric diseases [3]. Early life adversity could exert a direct effect on depression via biological systems, such as the hypothalamic pituitary axis, and these effects could be independent of exposure to adulthood adversity [23]. For example, the risk of depression in persons with multiple early-life adverse experiences is four times that of a person who has not experienced early-life trauma [10]. Among the general population, young adults are in particular vulnerable population to the MDD. However, there is still lack of studies focusing on relationship between ELA and depression in the young adults from the perspective of brain morphology.

Until now, whether ELA has influence on brain morphology is still in controversy; a number of studies show ELA-related hippocampal volume reductions in healthy populations $[8,28]$, whereas others did not reveal this finding [6]. Considering different models (primates vs. non-primates), different trauma occurring ages, as well as different comorbid diseases, the relationship between ELA and brain structure is far from clear conclusion $[1,16,22]$. As a result, we launched a study comparing the cerebral computed tomography (CT) imaging of young adult of MDD with or without ELA, so as to find the role of ELA in the brain structure of young adult suffered from MDD.

\section{MATERIALS AND METHODS}

We performed a clinical cross-sectional observational study and recruited 40 young adult male MDD patients from the Department of Psychiatry of No. 261
Hospital, PLA. All patients (aged between 18 and 26 years old) met the DSM-IV criteria for MDD confirmed by a structured interview conducted by two certified psychiatrists, and had a 17-item Hamilton Depression (HAMD) Scale score more than 17 points. All of the subjects were first-episode, treatment-naïve, then they were further divided into two groups according to whether they had an experience of ELA: MDD patients with ELA experience $(E+D)$ vs. MDD patients without ELA experience $(E-D)$. Subjects were excluded from the study if they had a history of alcohol or drug abuse, neurological or serious physical diseases (e.g., gastrointestinal, neurological, endocrine, or cardiovascular disorders).

Neuropsychiatric Inventory (NPI) was developed by Cummings et al. [7] to assess and quantify neurobehavioural disturbances in dementia patients. It has an Apathy subscale, which consists of a general screening item rated on a Yes/No basis. The overall frequency (1-4) and severity (1-3) are then rated. Scores on the NPI depression subscale range from 0 to 12 , with higher scores indicating more severe depression.

Early life adversity was quantified with the 28-item Chinese version-Childhood Trauma Questionnaire (CTQ), which assesses five types of adverse childhood experience subcomponents: emotional abuse (EA), physical abuse (PA), sexual abuse (SA), emotional neglect (EN), and physical neglect (PN). Scores ranged from 5 to 25 for each subscale, with high scores indicating strong exposure to the ELA [11]. According to the manual, patients who were positive for a history of childhood maltreatment based on mild to moderate cutoff scores of the total CTQ score (i.e., 41 points) [2]. In detail, cutoff scores according to Walker et al. [25] for each subscomponents were for sexual abuse $\geq 8$, physical abuse $\geq 8$, emotional abuse $\geq 10$, physical neglect $\geq 10$, and emotional neglect $\geq 15$.

Brain CT examinations were acquired on a 64-slice SIEMENS SOMATOM Definition Flash CT scanner. All $\mathrm{CT}$ images were performed with patients in the supine position, untilted, using the anatomical landmarks of the scull base and vertex. Slice thickness was $5 \mathrm{~mm}$. From each collected CT scan, two representative and consecutive images, displaying the third ventricles (TVs) at the level of the thalami in their largest dimensions were chosen. Maximum diameter of the TV and third ventricular volume were measured and calculated according to the methods of Wang's [26] automatically. 
Table 1. Overall characteristics of subjects

\begin{tabular}{|c|c|c|c|c|}
\hline & All $(n=40)$ & $E+D(n=21)$ & $E-D(n=19)$ & $\mathbf{P}$ \\
\hline Age [years] & $21.73(2.52)$ & $21.85(2.65)$ & $21.58(2.53)$ & NS \\
\hline Education level & $11.73(2.35)$ & $11.52(2.27)$ & $11.94(2.48)$ & NS \\
\hline HAMD & $22.40(4.24)$ & $23.42(4.75)$ & $21.26(3.36)$ & NS \\
\hline \multicolumn{5}{|l|}{ NPI-subscale } \\
\hline Delusions & $1.38(0.68)$ & $1.29(0.64)$ & $1.47(0.70)$ & NS \\
\hline Hallucinations & $1.45(1.06)$ & $1.47(1.08)$ & $1.42(1.07)$ & NS \\
\hline Agitation & $0.88(0.82)$ & $1.05(0.86)$ & $0.68(0.75)$ & NS \\
\hline Depression & $4.87(1.91)$ & $4.67(1.83)$ & $5.10(2.02)$ & NS \\
\hline Anxiety & $3.25(2.47)$ & $2.62(1.96)$ & $3.95(2.81)$ & NS \\
\hline Euphoria & $1.35(1.17)$ & $1.62(1.36)$ & $1.05(0.85)$ & NS \\
\hline Apathy & $4.25(2.34)$ & $4.61(2.54)$ & $3.84(2.09)$ & NS \\
\hline Disinhibition & $1.25(1.13)$ & $1.42(1.03)$ & $1.05(1.22)$ & NS \\
\hline Irritability & $1.73(1.22)$ & $1.71(1.27)$ & $1.74(1.20)$ & NS \\
\hline Aberrant motor behaviour & $1.70(1.26)$ & $1.71(1.35)$ & $1.68(1.20)$ & NS \\
\hline Nighttime behaviour disturbances & $3.10(2.76)$ & $2.95(2.50)$ & $3.26(3.08)$ & NS \\
\hline Appetite and eating abnormalities & $3.63(2.52)$ & $3.62(2.69)$ & $3.63(2.39)$ & NS \\
\hline
\end{tabular}

NS - not statistically significant

Table 2. Childhood Trauma Questionnaire (СТО) scores of subjects

\begin{tabular}{lcccc}
\hline & All $(\mathbf{n}=\mathbf{4 0})$ & $\mathbf{E}+\mathbf{D}(\mathbf{n}=\mathbf{2 1})$ & $\mathbf{E}-\mathbf{D}(\mathbf{n}=\mathbf{1 9})$ & $\mathbf{P}$ \\
\hline CT0 & $39.88(5.98)$ & $43.76(4.79)$ & $35.58(3.83)$ & 0.000 \\
EA & $8.63(3.19)$ & $9.38(3.56)$ & $7.79(2.57)$ & 0.116 \\
PA & $7.30(1.90)$ & $7.71(2.28)$ & $6.84(1.26)$ & 0.140 \\
SA & $6.10(1.13)$ & $6.24(1.09)$ & $5.95(1.17)$ & 0.423 \\
EN & $8.65(3.42)$ & $9.57(4.01)$ & $7.63(2.31)$ & 0.067 \\
PN & $9.78(4.36)$ & $11.33(5.33)$ & $8.05(1.93)$ & 0.014 \\
\hline
\end{tabular}

EA — emotional abuse; PA — physical abuse; SA — sexual abuse; EN — emotional neglect; PN — physical neglect

\section{Statistical analysis}

Student's t-test was used to compare the HAMD scores, NPI scores, CTQ subcomponents scores, TV width and volume of patients from E+D and E-D groups. Bivariate correlation analysis was used to find the association of TV width/volume and total CTQ score/CTQ subcomponent scores. The data are expressed as the mean \pm standard error. $P$ value less than 0.05 was considered statistically significant. All statistical analyses were carried out using the SPSS, 17.0 statistical software package (IBM Corp., Armonk, New York, USA).

\section{RESULTS}

There were no significant differences across groups with regards to age and years of education.
There were no significant differences across groups in total depression scores of HAMD and NPI-items ( $p>0.05$ ). Scoring of total CTQ and PN scores differed between groups $(p<0.001$ and $p=0.016$ ), while other subcomponent scores did not show significant differences (Tables 1, 2).

The mean \pm standard deviation $(\mathrm{SD})$ total ventricle width were $0.077 \pm 0.122 \mathrm{~cm}$ and $0.697 .08 \pm$ $\pm 0.759 \mathrm{~cm}$ in $E+D$ and $E-D$ patients $(p=0.037)$, and the mean $( \pm S D)$ total ventricle volumes were $1.587 \pm$ $\pm 0.432 \mathrm{~cm}^{3}$ and $1.193 \pm 0.331 \mathrm{~cm}^{3}$ in $E+D$ and $E-D$ patients $(p=0.003)$, respectively. This finding revealed that TV enlargement was more obvious in $E+D$ compared with E-D. The examples were showed in Figure 1. Bivariate correlation analysis was done 


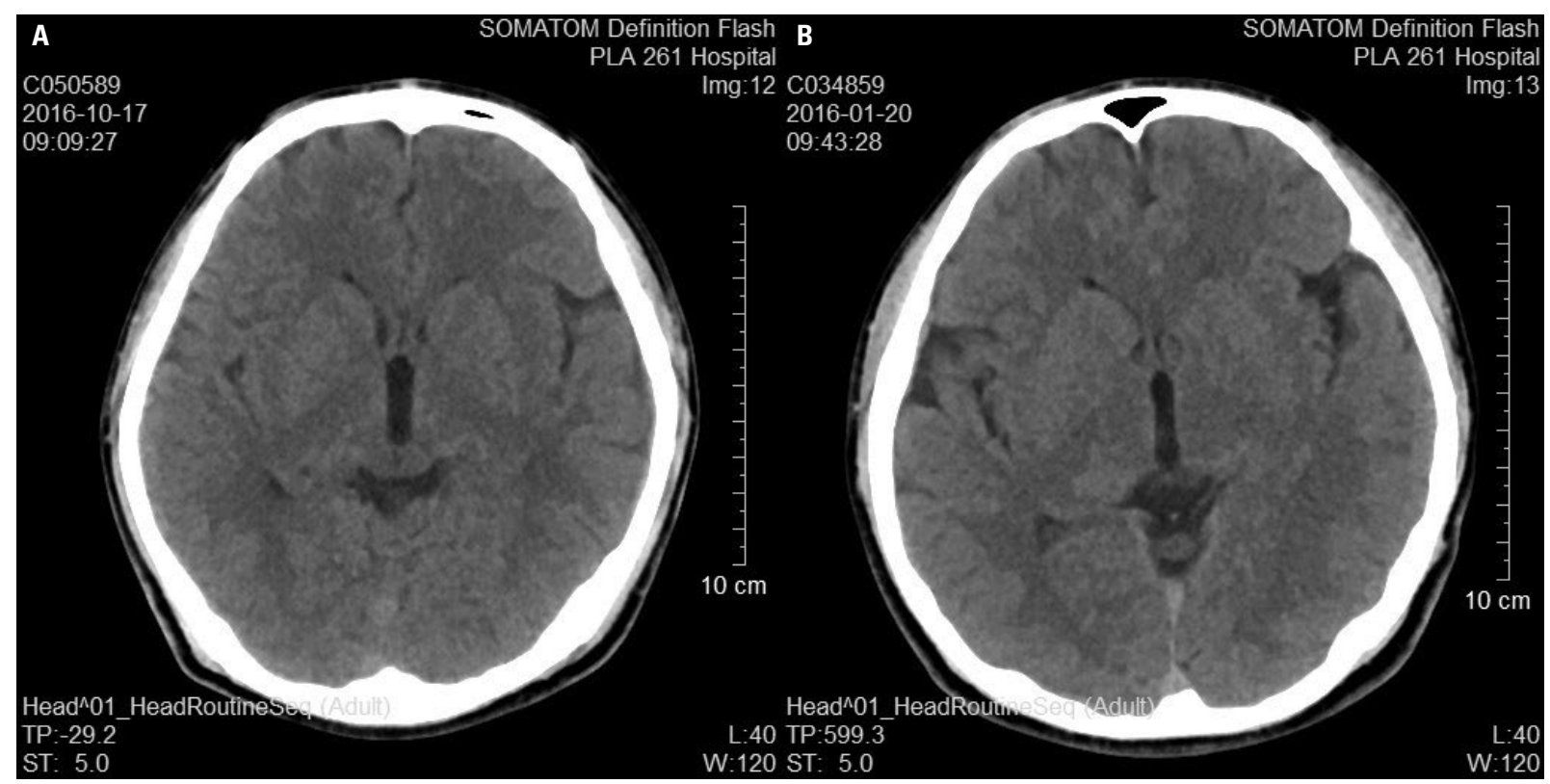

Figure 1. Example of computed tomography scan image from $E+D$ patients $(\mathbf{A})$ and $E-D$ patients $(\mathbf{B})$.

to find the correlation between CTQ subcomponent scores and TV width/volume. Among all the subcomponents, PN score had a mild correlation width TV volume significantly $(p=0.02, r=0.36)$, while, there is still a trend between PN score and TV width; however, the data did not reach significance $(p=0.39)$.

\section{DISCUSSION}

In current study, we found that in adolescents and young adults suffering from MDD, those patients who experienced childhood traumatic events showed more serious TV enlargement; furthermore, TV volume was associated with ELA severity positively more obviously. Furthermore, of Chinese version-CTQ subscales, TV volume, rather than enlargement, showed positive correlation with PN.

Traumatic experiences in childhood can be found in many patients of depression [4]. In a cross-sectional study launched by Negele et al. [19], clinically significant histories of childhood trauma could be reported in as high as $75.6 \%$ of chronic depression patients. For those with MDD, a recent study in the United States of America found that nearly $62.5 \%$ patients reported more than two traumatic events [27]. In our study, we found $47.5 \%$ patients of MDD were ELA positive assessed by Chinese version-CTQ. The discrepancy might lie in two aspects, cultural difference and sample size.
Increased volumetric changes seen in the TV and changes in other closely associated structures are hypothesised as a basic anatomical characteristic of depression [15]. As we have mentioned previously, hypothalamic pituitary axis which was related to ELA and took part in MDD aetiology, also belonged to the structure of TV. In light of the Hendrie's theory, animals as well as human beings have to adapt the potential dangerous situations when they run into attack stimuli. This adaptation is undermined by the anatomical changes of structures around the TV, reflected by the series of clinical symptoms, such as hunched posture, avoidance of eye contact, loss of appetite for food and libido and sleep disruption, etc. [15]. In present study, TV width and volume were significantly larger in MDD patients with ELA experience than those without ELA experience. This finding could be explained by the childhood trauma. We proposed that ELA induced the atrophy of structures around TV causing TV enlargement and MDD vulnerability.

More in detail, bivariate correlation analysis showed that width and volume of TV were positively correlated with CTQ total ( $p=0.037$ vs. $p=0.003$ ). For CTQ subcomponent scales, PN score correlated with volume of TV obviously ( $p=0.02, r=0.362)$, there is still a trend between PN score and TV width; however, the data did not reach significance $(p=0.396)$. 
In 2013, a study from another group from China demonstrated that brain white matter densities in the bilateral inferior parietal lobe negatively correlated with neglect total scores on the CTQ [20]. In their article, authors used PN score (cutoff point $=10$ ) or EN score (cutoff point $=15$ ) or both (cutoff point $=20$ for comorbid EN and PN), rather than abuse scores as factors that affected structure and function of specific brain areas related to sensation, perception, cognition, and affective modulation. In our study, our results revealed once again, that $P N$ is an important factor for brain structure of MDD patients. Both results implied that neglect injury (emotional or physical) during childhood might be the most likely element for a child, which could do harm to brain development and result in affective disorders finally. Considering the development style of developing countries, we need to pay more attention to living condition of the left-behind children and orphans in modern society.

\section{Limitations of the study}

There are some limitations in our study. First, we only collected a total of 40 patients. The limited number of subjects and data limited a more detailed analysis. Second, we chose to exclude female patients from our study, and one advantage of this is prevention of gender bias. Actually, there are more women than men who suffered from MDD.

\section{CONCLUSIONS}

In conclusion, ELA was common in young adult male patients suffering from MDD; those who have a history of childhood trauma show larger TV width and volume. Among CTQ subcomponents, PN correlated significantly with volume of TV. All these findings might serve as a good reference for the aetiology and pathology of ELA in MDD patients.

\section{REFERENCES}

1. Baker LM, Williams LM, Korgaonkar MS, et al. Impact of early vs. late childhood early life stress on brain morphometrics. Brain Imaging Behav. 2013; 7(2): 196-203, doi: 10.1007/s11682-012-9215-y, indexed in Pubmed: 23247614

2. Bernstein D, Fink L. Childhood trauma questionnaire: a retrospective self-report questionnaire and manual. San Antonio, TX: Psychological Corp. 1998.

3. Carballedo A, Lisiecka D, Fagan A, et al. Early life adversity is associated with brain changes in subjects at family risk for depression. World J Biol Psychiatry. 2012; 13(8):
569-578, doi: 10.3109/15622975.2012.661079, indexed in Pubmed: 22515408.

4. Caspi A, Sugden K, Moffitt TE, et al. Influence of life stress on depression: moderation by a polymorphism in the 5-HTT gene. Science. 2003; 301(5631): 386-389, doi: 10.1126/ science.1083968, indexed in Pubmed: 12869766.

5. Chapman DP, Liu Y, Presley-Cantrell LR, et al. Adverse childhood experiences and frequent insufficient sleep in 5 U.S. States, 2009: a retrospective cohort study. BMC Public Health. 2013; 13: 3, doi: 10.1186/1471-2458-13-3, indexed in Pubmed: 23286392.

6. Cohen RA, Grieve S, Hoth KF, et al. Early life stress and morphometry of the adult anterior cingulate cortex and caudate nuclei. Biol Psychiatry. 2006; 59(10): 975-982, doi: 10.1016/j.biopsych.2005.12.016, indexed in Pubmed: 16616722.

7. Cummings JL, Mega M, Gray K, et al. The Neuropsychiatric Inventory: Comprehensive assessment of psychopathology in dementia. Neurology. 1994; 44(12): 2308-2308, doi: 10.1212/wnl.44.12.2308.

8. Dannlowski U, Stuhrmann A, Beutelmann V, et al. Limbic scars: long-term consequences of childhood maltreatment revealed by functional and structural magnetic resonance imaging. Biol Psychiatry. 2012; 71(4): 286-293, doi: 10.1016/j.biopsych.2011.10.021, indexed in Pubmed: 22112927.

9. Espejo EP, Hammen CL, Connolly NP, et al. Stress sensitization and adolescent depressive severity as a function of childhood adversity: a link to anxiety disorders. J Abnorm Child Psychol. 2007; 35(2): 287-299, doi: 10.1007/s10802006-9090-3, indexed in Pubmed: 17195949.

10. Felitti VJ, Anda RF, Nordenberg D, et al. Relationship of childhood abuse and household dysfunction to many of the leading causes of death in adults. The Adverse Childhood Experiences (ACE) Study. Am J Prev Med. 1998; 14(4): 245-258, indexed in Pubmed: 9635069.

11. Fu WQ, Yao SQ. Initial Reliability and validity of childhood trauma questionnaire (CTQ-SF) applied in Chinese college students. Chin J Clin Psychol. 2005; 13(1): 40-42.

12. Gillespie C, Nemeroff C. Corticotropin-Releasing factor and the psychobiology of early-life stress. Curr Dir Psychol Sci. 2016; 16(2): 85-89, doi: 10.1111/j.14678721.2007.00481.x.

13. Heim C, Binder EB. Current research trends in early life stress and depression: review of human studies on sensitive periods, gene-environment interactions, and epigenetics. Exp Neurol. 2012; 233(1): 102-111, doi: 10.1016/j. expneurol.2011.10.032, indexed in Pubmed: 22101006.

14. Heim C, Plotsky PM, Nemeroff CB. Importance of studying the contributions of early adverse experience to neurobiological findings in depression. Neuropsychopharmacology. 2004; 29(4): 641-648, doi: 10.1038/sj.npp.1300397, indexed in Pubmed: 15034558.

15. Hendrie CA, Pickles AR. Depression as an evolutionary adaptation: anatomical organisation around the third ventricle. Med Hypotheses. 2010; 74(4): 735-740, doi: 10.1016/j.mehy.2009.10.026, indexed in Pubmed: 19931308.

16. Hodel AS, Hunt RH, Cowell RA, et al. Duration of early adversity and structural brain development in post-institu- 
tionalized adolescents. Neuroimage. 2015; 105: 112-119, doi: 10.1016/j.neuroimage.2014.10.020, indexed in Pubmed: 25451478.

17. Hussey JM, Chang JJ, Kotch JB. Child maltreatment in the United States: prevalence, risk factors, and adolescent health consequences. Pediatrics. 2006; 118(3): 933-942, doi: 10.1542/peds.2005-2452, indexed in Pubmed: 16950983.

18. Lewis AJ, Olsson CA. Early life stress and child temperament style as predictors of childhood anxiety and depressive symptoms: findings from the longitudinal study of Australian children. Depress Res Treat. 2011; 2011: 296026, doi: $10.1155 / 2011 / 296026$, indexed in Pubmed: 22203890.

19. Negele A, Kaufhold J, Kallenbach L, et al. Childhood Trauma and Its Relation to Chronic Depression in Adulthood. Depress Res Treat. 2015; 2015: 650804, doi: 10.1155/2015/650804, indexed in Pubmed: 26693349.

20. Peng $H$, Ning $Y$, Zhang $Y$, et al. White-matter density abnormalities in depressive patients with and without childhood neglect: a voxel-based morphometry (VBM) analysis. Neurosci Lett. 2013; 550: 23-28, doi: 10.1016/j. neulet.2013.06.048, indexed in Pubmed: 23831354.

21. Scher CD, Forde DR, McQuaid JR, et al. Prevalence and demographic correlates of childhood maltreatment in an adult community sample. Child Abuse Negl. 2004; 28(2): 167-180, doi: 10.1016/j.chiabu.2003.09.012, indexed in Pubmed: 15003400.

22. Spies G, Ahmed-Leitao F, Fennema-Notestine C, et al. Effects of HIV and childhood trauma on brain morphometry and neurocognitive function. J Neurovirol. 2016; 22(2): 149-158, doi: 10.1007/s13365-015-0379-2, indexed in Pubmed: 26424107.
23. Stansfeld SA, Clark C, Rodgers B, et al. Repeated exposure to socioeconomic disadvantage and health selection as life course pathways to mid-life depressive and anxiety disorders. Soc Psychiatry Psychiatr Epidemiol. 2011; 46(7): 549-558, doi: 10.1007/s00127-010-0221-3, indexed in Pubmed: 20383489.

24. The World Bank. The Global Burden of Disease and Risk Factors. Washington, D.C.. The international Bank for Reconstruction and Development/The World Bank; New York: Oxford University Press. 2006.

25. Walker EA, Unutzer J, Rutter C, et al. Costs of health care use by women $\mathrm{HMO}$ members with a history of childhood abuse and neglect. Arch Gen Psychiatry. 1999; 56(7): 609-613, indexed in Pubmed: 10401506.

26. Wang J, Xiang Z, Zhan Y, et al. Multi-slice spiral ct the third and fourth ventricular volume measurement and its clinical significance. Chin J Med Guide. 2011; 13(6): 998$-999$.

27. Williams LM, Debattista C, Duchemin AM, et al. Childhood trauma predicts antidepressant response in adults with major depression: data from the randomized international study to predict optimized treatment for depression. Transl Psychiatry. 2016; 6: e799, doi: 10.1038/tp.2016.61, indexed in Pubmed: 27138798.

28. Woon FL, Sood S, Hedges DW. Hippocampal volume deficits associated with exposure to psychological trauma and posttraumatic stress disorder in adults: a meta-analysis. Prog Neuropsychopharmacol Biol Psychiatry. 2010; 34(7): 1181-1188, doi: 10.1016/j.pnpbp.2010.06.016, indexed in Pubmed: 20600466.

29. Zhao XF, Zhang YL, Li LF. Childhood abuse: an investigation of 435 middle school students. Chin J Clin Psychol. 2004; 12(4): 377-379. 